\title{
PERIODIC TRENDS IN TWO-PHASE FLOW THROUGH A VERTICAL MINICHANNEL: WAVELET AND MULTISCALE ENTROPY ANALYSES BASED ON DIGITAL CAMERA DATA
}

\author{
Grzegorz GÓRSKI*, Grzegorz LITAK ${ }^{* *+*}$, Romuald MOSDORF*, Andrzej RYSAK ${ }^{* *}$ \\ g.gorski@pb.edu.pl, g.litak@pollub.pl, r.mosdorf@pb.edu.pl, a.rysak@pollub.pl
}

\begin{abstract}
*Faculty of Mechanical Engineering, Bialystok University of Technology, ul. Wiejska 45C, 15-351, Bialystok, Poland
${ }^{*}$ Faculty of Mechanical Engineering, Lublin University of Technology, ul. Nadbystrzycka 36, 20-618 Lublin, Poland

${ }^{* *}$ Department of Process Control, AGH University of Science and Technology, ul. Mickiewicza 30, 30-059 Krakow, Poland
\end{abstract}

received 24 March 2018, revised 15 March 2019, accepted 21 March 2019

\begin{abstract}
By changing the air and water flow relative rates in the two-phase (air-water) flow through a minichannel, we observe aggregation and partitioning of air bubbles and slugs of different sizes. An air bubble arrangement, which show non-periodic and periodic patterns. The spatiotemporal behaviour was recorded by a digital camera. Multiscale entropy analysis is a method of measuring the time series complexity. The main aim of the paper was testing the possibility of implementation of multiscale entropy for two-phase flow patterns classification. For better understanding, the dynamics of the two-phase flow patterns inside the minichannel histograms and wavelet methods were also used. In particular, we found a clear distinction between bubbles and slugs formations in terms of multiscale entropy. On the other hand, the intermediate region was effected by appearance of both forms in non-periodic and periodic sequences. The preliminary results were confirmed by using histograms and wavelets.
\end{abstract}

Key words: digital camera data, two-phase flow, patterns identification, multiscale entropy, wavelets

\section{INTRODUCTION}

Two-phase dynamics is a common issue in many technical minichannel systems of various diameters, ranging from micro- to centimetres. Different properties of the flowing phases lead to the main problems with pressure and temperature control during flows. That problems motivated many works on patterns identification of two-phase flow in minichannels from measurements or modelling (Zhao and Rezkallah, 1993; Wongwises and Pipathattakul, 2006; Chen et al., 2006; Zong et al., 2010; Anjos et al., 2014). More advanced studies on flow patterns were conducted by Wang et al. (2003), who used Hurst and Lyapunov exponents, as well as the correlation dimension. Simultaneous to that investigations, Jin et al. (2003) used the correlation dimension and, additionally, the Kolmogorov entropy. Later, Mosdorf et al. (2005) provided the results of non-linear approaches to the temperature and pressure fluctuations in microchannels. More recently, recurrence statistics were performed to the two-phase flows (Gorski et al., 2015a; 2015b; 2016) by employing a laser-phototransistor light transitivity sensor. These investigations enable to identify the transitions from bubbles to slugs and churn, and conclude on corresponding patterns stabilities. Multiscale character of flows were raised by Fan et al. $(2013,2015)$, who also used the data of light transmission through a minichannel. Finally, the complex multiscale morphological analysis using electric conductance signals was proposed by Lian et al. (2016).

The main aim of the paper was testing the possibility of implementation of multiscale entropy for the two-phase flow patterns' classification. For better understanding, the dynamics of twophase flow patterns inside the minichannel histograms and wave- let methods were also used. The concepts of wavelets and multiscale entropy applied directly to the digital camera snapshots (Rysak et al., 2016).

\section{EXPERIMENTAL SETUP}

We performed the measurements and analysed the related data recorded for different flow patterns (water-air at $21^{\circ} \mathrm{C}$ ) in a circular channel having a diameter of $2 \mathrm{~mm}$. In Fig. 1, the schema of experimental stand is presented. In the conditions of the small diameter minichannel, a special generator of mini bubbles was designed (8). The proportional pressure regulator (Metal Work Regtronic with an accuracy of $1 \mathrm{kPa}$ ) was used to maintain the constant overpressure in the supply tank (10 - Fig. 1) - the overpressure was $50 \mathrm{kPa}$. Flow patterns were recorded using the Phantom v. 1610 digital camera at $5000 \mathrm{fps}(1280 \times 64$ pixels $)$. The amount of air flowing through the minichannel was also measured by laser-phototransistor sensor (3). Data from the sensors was acquired by the acquisition system (Data translation 9804 , an accuracy of $1 \mathrm{mV}$ for voltages in the range of $-10 \mathrm{~V}$ to 10 V), (11) at a sampling rate of $5 \mathrm{kHz}$.

The measurement results are presented in Fig. 2 (camera snapshots) for changing air volume flow rate $q_{a}\{0: 00632,0.1\}$ $\mathrm{I} / \mathrm{min}$ and changing water volume flow rate $q$. Note that air bubbles were produced with different shapes depending on the airflow $\mathrm{q}_{\mathrm{a}}$. In the limit of small $q_{a}$ and large $q$ (Fig. 2a), the air bubbles are of fairly small and variable sizes. Their sizes are increasing with decreasing $q$ (Fig. 2d). On the other hand, for larger $\left(q_{a}\right)$, decreasing $\mathrm{q}$ leads to stabilizing of air slugs (elongated bubbles) - see Figs. 3a-d. 


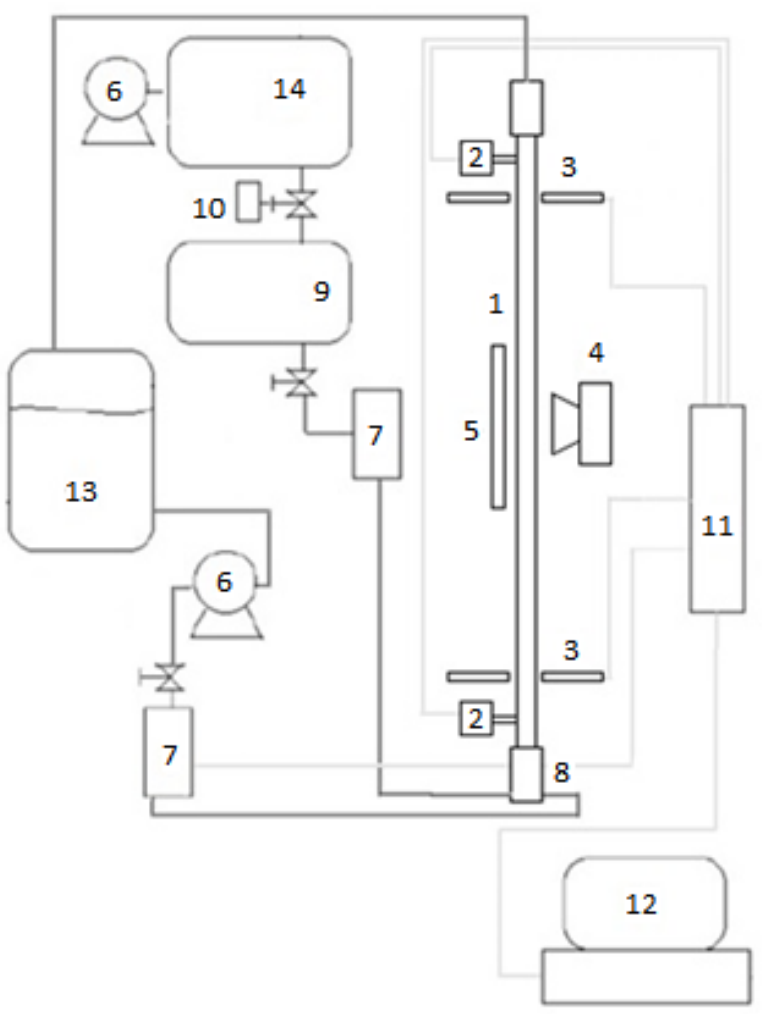

Fig. 1. Experimental setup: 1 - vertical minichannel with a diameter of 3 $\mathrm{mm}, 2$ - pressure sensors (MPX12DP), 3 - laser-phototransistor sensor, 4 - Phantom v. 1610 camera, 5 - lighting, 6 - pumps (air or water), 7 - flow meters, 8 - mini bubbles generator, 9 - air tank, 10 - automatic valve to maintain a constant pressure in the tank 9, 11 - data acquisition station (DT9800), 12 - computer, 13 - water tank, 14 - air tank

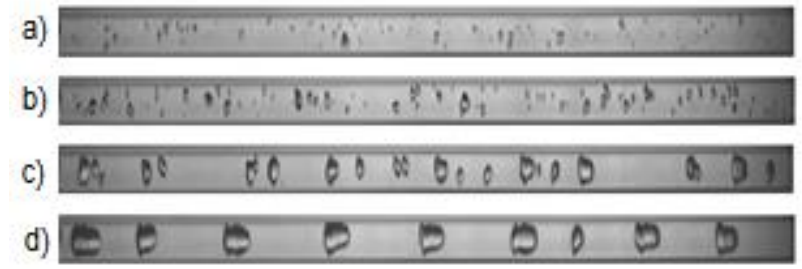

Fig. 2. Photos of the selected flow cases with increasing water volume flow rate $q[/ / \mathrm{min}]: 0.234(\mathrm{a}), 0.173(\mathrm{~b}), 0.091$ (c), and 0.027 (d), respectively. The air volume flow rate was fixed to $q_{a}=0: 00632 \mathrm{l} / \mathrm{min}$.

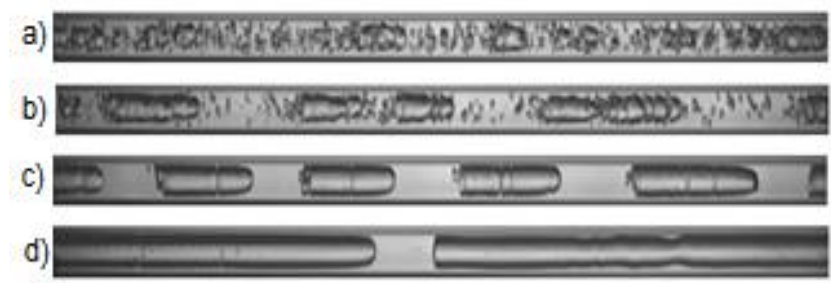

Fig. 3. Photos of the selected flow cases with increasing water volume flow rate $q[\mathrm{l} / \mathrm{min}]: 0.216(\mathrm{a}), 0.145(\mathrm{~b}), 0.071$ (c), and $0.012(\mathrm{~d})$, respectively. The air volume flow rate was fixed to $q_{a}=0.1 \mathrm{l} / \mathrm{min}$.

Following the pioneering image processing techniques (Otsu, 1979; Haralick et al., 1987), we explore the digital camera signal obtained from the bubbles and slugs contours passing through the cross-sectional gate observation (Rysak et al., 2016). Note that in the previous attempts of digital conversion, the contrast was introduced between different phases (Mydlarz-Gabryk et al., 2014; Riano et al., 2015; Ansari and Azadi, 2016). Here, we present the alternative and simpler way with processing pixels of contours between two flowing phases.
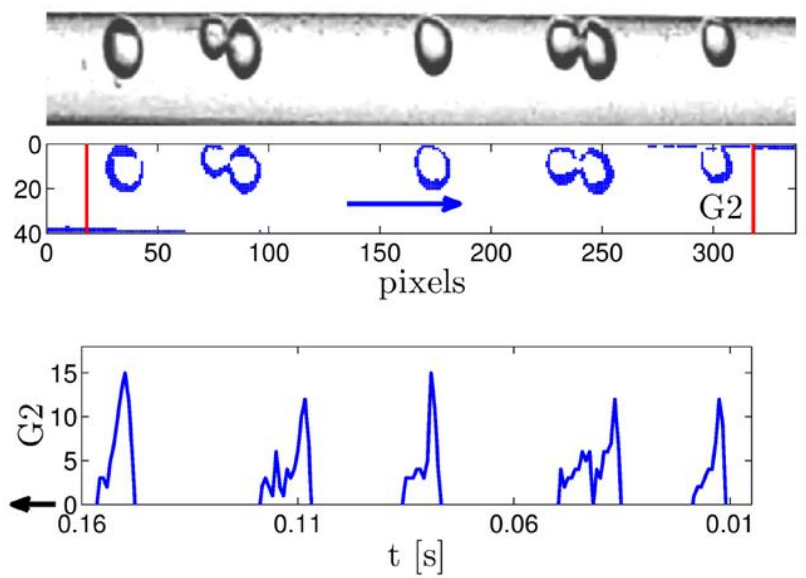

Fig. 4. The digital camera signal was obtained from the bubbles and slugs contours passing through the cross-sectional gate observation G2 (Rysak et al., 2016). Note difference in the horizontal axis orientation.

The contour frames were obtained by converting filtered images to $0-1$ values matrices. One of the captured image frames of the two-phase flow is shown in the upper part in Fig. 4. The middle figure shows its contour frame representation. The red line on the right hand side indicates the gate $\mathrm{G} 2$, where pixels are counted. The movement of the mixture takes place to the right (arrow). With the laps of time, the structures of the gas flowing through the gate $\mathrm{G} 2$ build a time series (as shown in the bottom image in Fig. 4). Consequently, we observe the time series in terms of photo pixels passing through the defined gate.

\section{DIGITAL TIME SERIES ANALYSIS}

The results of the resulting time series corresponding to the cases presented in Figs. 2 and 3 are presented in Figs. 5 and 6 , respectively. Note that $q_{a}=0$ would correspond to single phase (water) flow. Most of the time series looks random (Fig. 5). However, the pattern is changing from single lines representing small bubbles in Fig. 5a and $5 \mathrm{~b}$ to longer slugs in Fig. $5 \mathrm{~d}$. On the way, in Fig. $5 c$, there is a mixture of these formations, which looks like a chaotic transient (Sen et al., 2008). On the other hand, the longest stable level of 10 pixels corresponds to the slug flow (as shown in Fig. 6 at the lower panel [b]).

Interestingly, one can observe the interesting periodic intermittent (water-air) flow (see Fig. 6 at the middle panels). This is a spatio-temporal self-organization phenomenon of the flow determined by the air-water mixture of fluid and the flow conditions (see Fig. 6c): the flow rates, their viscosity, and the geometry of minichannel. I believe it should be well imitated, which corresponds to a convergence in the bubbles sizes. However, the phase variations (Fig. 6c) confirm that the periodic tendencies are not fully developed and the time series are characterised by only short time correlations. 


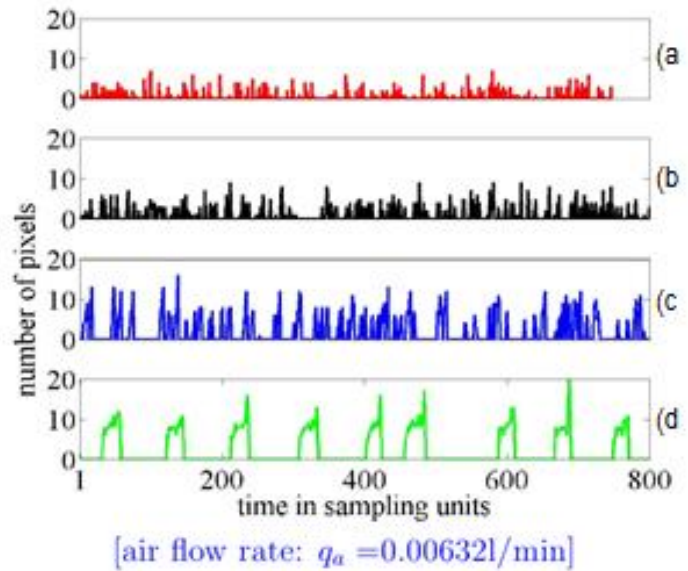

Fig. 5. Time series in terms of number of pixels xi passing through the gate in the central cross-section of the camera view (Fig. 1). The cases presented downward correspond to (a)-(d) in Fig. 2. The sampling time step was $0.2 \times 10^{-3} \mathrm{~s}$. Number of points (range of horizontal axis) was 800 .

\section{PIXEL STATISTICAL DISTRIBUTIONS - HISTOGRAMS}

Distributions of pixels registered by the digital camera can be investigated by histograms. Interestingly, we observe the evolution from the system of air bubbles' deficiency to larger bubbles, and finally the elongated bubbles - slugs. In case of the low amount of air flow $q_{a}=0.00632 \mathrm{l} / \mathrm{min}$, the small air bubbles are noticeable in a large water space (Fig. 2a-b).

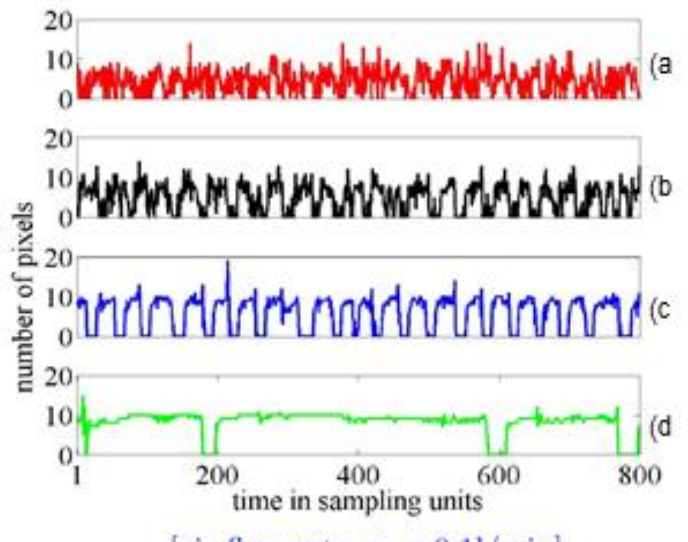

[air flow rate: $q_{a}=0.11 / \mathrm{min}$ ]

Fig. 6. Time series in terms of number of pixels xi passing through the gate in the central cross-section of the camera view (Fig. 1). The cases presented downward correspond to (a)-(d) in Fig. 3, respectively. The sampling time step was $0.2 \times 10^{-3} \mathrm{~s}$. Number of points (range of horizontal axis) was 800 .
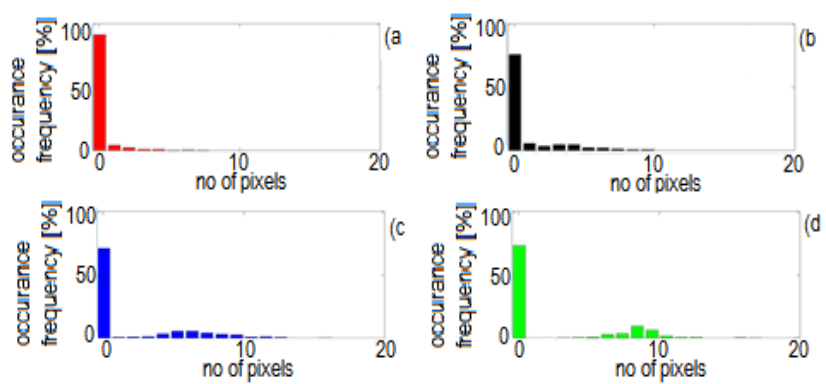

Fig. 7. Histograms for the cases presented in Fig. 2
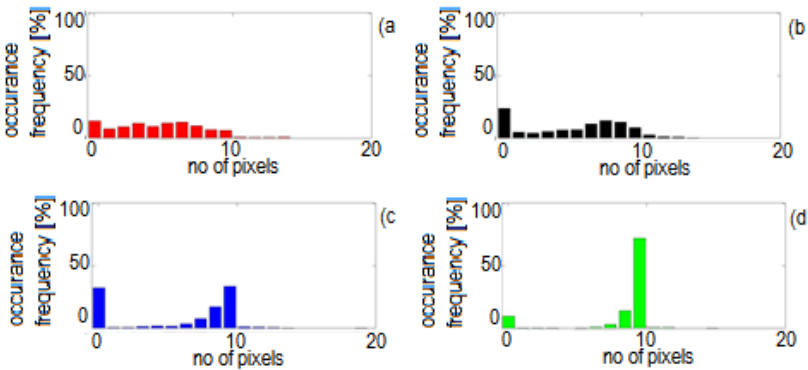

Fig. 8. Histograms for the cases presented in Fig. 3

Consequently, these cases are accompanied by a fairly large maximum in histograms with 0 pixel (Figs. 7a, b), which is clearly visible. By decreasing the water flow, we observe two maxima in histograms (Figs. 7c, d) with continued domination of 0 pixel peak. Finally, for higher amount of air flow, the 0 pixel probability peak is strongly reduced (Figs. 8a-8d). Additionally, the cases in Figs. 8c, $d$, with the strong increase in the longer length occurrence frequency of pixel numbers, reflects the presence of slugs.

\section{WAVELET ANALYSIS}

A statistical approach of the pixels counting presented in the last section gave the idea of the distribution of bubbles and slugs in terms of the appearance of their characteristic lengths. On the other hand, bubbles and slugs can appear in a random way or with a given periodicity. To clarify this point, we decided to use continuous wavelets, which can distinguish the short interval periodic self-organizing structures. This is an alternative method to the earlier proposed recurrence studies (Gorski et al., 2015b), which were conducted for laser-photoresistor transitivity data (Gorski et al., 2015b).

In the current study, to show the emerging periods, we present the wavelet analysis of the results. We study the time series of pixels $N p(t)$ given by the continuous wavelet transformation (CWT) (Kumar and Foufoula-Georgiou, 1997; Torrence and Compo, 1998; Lonkwic et al., 2017). The corresponding continuous wavelet transform with respect to the wavelet function $\psi($.$) is$ defined as follows:

$W_{s, n}(N p)=\sum_{i=1}^{N} \frac{1}{s} \psi\left(\frac{i-n}{s}\right) \frac{\left(N p\left(t_{i}\right)-\langle N p\rangle\right)}{\sigma_{N p}}$,

where: $\langle N p\rangle$ and $\sigma N p$ are the averages and standard deviations of pixel number registered, respectively. Finally, the wavelet $\psi($.$) is referred to as the mother wavelet, and the letters s$ and $n$ denote the scale and the time indices, respectively. The wavelet power spectrum (WPS) of the $N p$ time series is defined as the square modulus of the CWT:

$P_{w}=\left|W_{s, n}\right|^{2}$.

In the present calculations, we used a complex Morlet wavelet as the mother wavelet. The Morlet wavelet consists of a plane wave modulated by a Gaussian function and it is described by:

$\psi(\eta)=\pi^{-1 / 4} \mathrm{e}^{\mathrm{i} \theta_{0} \eta} \mathrm{e}^{-\eta^{2} / 2}$,

where: $\theta_{0}$ is the centre frequency, also referred to as the order of the wavelet, and $\eta$ is a renormalized time variable. Namely, $\theta_{0}$ defines the number of oscillations in the wavelet and thus controls the time/frequency resolutions. In our analysis, we used $\theta_{0}=6$, 
which provides a good balance between the time and frequency resolutions. Also, for the a.m. choice, the scale is approximately equal to the period, and therefore, the terms scale and period can be interchanged for interpreting the results. For general discussion, see: Kumar and Foufoula-Georgiou (1997), Torrence and Compo (1998) and Lonkwic et al. (2017). The corresponding results of CWT as the wavelet power spectra are presented in Figs. 9 and 10. Using these techniques, it was possible to select the regions of periodic formations. On the logarithmic colour scale, these regions are shown by the dark red-brown colour. For instance, Figs. $10 \mathrm{~b}$ and $10 \mathrm{c}$ show clear periods with around 35 sampling times. A less spectacular period of about 80 sampling times is also visible in Fig. 9d. Other cases are more random showing much shorter periods.
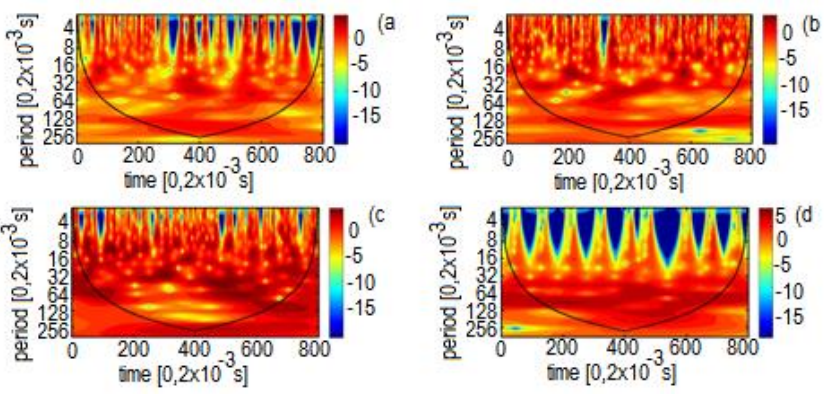

Fig. 9. Wavelets for the cases presented in Fig. 2. Colours, from blue to red, corresponds to increase in the wavelet power. The black U-like curve limit the internal region of importance.
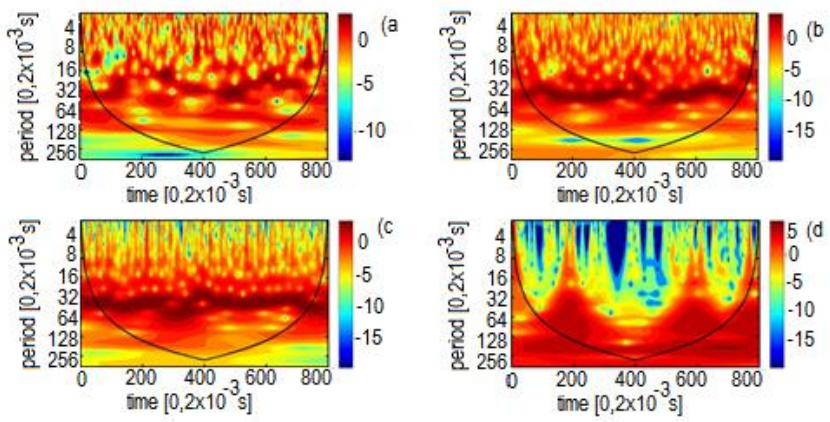

Fig. 10. Wavelets for the cases presented in Fig. 3. Colours, from blue to red, corresponds to increase in the wavelet power. The black U-like curve limit the internal region of importance.

\section{MULTISCALE ENTROPY}

To improve the understanding of the behaviour of complex systems that manifest themselves in non-linear behaviour, the multiscale entropy (Costa et al., 2003; 2005) analysis is becoming increasingly more popular (see: Borowiec et al., 2010; Litak et al., 2011; Wu et al., 2013, Borowiec et al., 2014). This method provides, for measured signals, a relative level of complexity of finite length time series. Unfortunately, there is no consensus of the complexity definition, but it is combined with 'meaningful structural richness' (Grassberger, 1991) contained over multiple spatiotemporal correlations.

The concept of multi-scale entropy (MSE) (Costa et al., 2003, 2005 ) is based on the coarse-graining procedure that uses a coarse-grained time series, as an average of the original data points within non-overlapping windows by increasing the scale factor $\mathrm{T}$ according to the following formula (see the cases $\tau=2$ and $n$ in Fig. 11):

$x_{j}^{(\tau)}=\frac{1}{\tau} \sum_{i=(j-1) \tau+1}^{j \tau} x_{i}$,

where $x_{i}$ is a raw one-dimensional acceleration time series $x=\left\{x_{1}, x_{2}, \ldots, x_{N}\right\}$. In this approach for each scale factor $\tau$, the MSE calculation based on the time series of the coarsegrained $x_{j}(\tau)$ :

$\operatorname{MSE}(x, \tau, n, r)=\operatorname{SampEn}\left(x^{(\tau)}, m, r\right)$,

where $m=2$ is the pattern length and $r$ is the similarity (tolerance) criterion and is usually chosen to be $r<\sigma(x),{ }^{[25]}$ here $\sigma(x)$ is the standard deviation of the original time series and $x(1) i=x_{i}$.

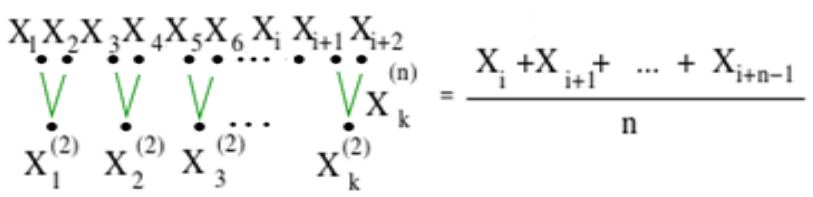

Fig. 11. Schema of multiscale entropy calculation. Here, $X_{i}$ denotes the i-th value on the time series shown in Fig. 5.

To estimate $\operatorname{SampEn}\left(x^{(\tau)}, m, r\right)$ from Eq. 6 (see also Fig. 11), we count the number of vector pairs denoted by $x^{(\tau)}{ }_{i}$ and $x^{(\tau)}{ }_{j}$ in the time series of length $m$ and $m+1$ having distance $\mathrm{d}\left[x^{(\tau)}{ }_{i}, x^{(\tau)}{ }_{j}\right]<r$. We denote them by $P_{m}$ and $P_{m+1}$, respectively. Finally, we define the sample entropy to be Richman and Moorman (2000):

$\operatorname{SampEn}\left(x^{(\tau)}, m, r\right)=-\log \frac{P_{m+1}}{P_{m}}$.

Strictly speaking, it is the minus of the logarithm of the conditional probability that the two sequences with a tolerance $r$ form points that remain within $r$ of each other at the next point. Continuing the research activity, Wu et al. (2013) introduced the concept of a composite multi-scale entropy (CMSE), which for higher scale factor provides entropy more reliably than the usual multiscale entropy by including multiple [k] combinations of neighbour points. The prescribed algorithm for CMSE calculations is the following formula:

$\operatorname{CMSE}(x, \tau, m, r)=\frac{1}{\tau} \sum_{k=1}^{\tau} \operatorname{SampEn}\left(x^{(\tau)}[k], m, r\right) .(7)$

The results of the complexity measure in terms of CMSE estimations for all the time series of our consideration (Figs. 5a, b) are presented in Fig. 12 for smaller $q_{a}$ and in Fig. 13 for larger $q_{a}$. All of the results indicate rather non-periodic character of the flow phenomenon. However, comparing the results (Figs. 13a13c) with the associated photos (see Figs. 3a-3c) and time series in Figs. 6a-6c), we can draw conclusions that complexity (expressed by turbulence flow) level is the highest at Fig. 13b. This is the intermediate region of coexistence of bubbles and slugs. Note also that Figs $12 \mathrm{a}-12 \mathrm{~d}$ show a similar structure with shifted maximum to the right hand side for decreasing $q_{a}$. This change is related to the size of bubbles (and possibly their velocities) appearing in the flow (Fig. 2). In contrast, Figs. 13a-13d show a spectacular transition, which is also visible in Fig. 3 . The bubble like flow is experiencing qualitative metamorphosis and the 
bubbles flow is changing to the slugs flow. In the entropy picture, this leads to the change of negative to positive inclination (compare Figs. 13a to 13d). Such an inclination could be a signal of oversampling (Borowiec et al., 2010); however, in our case, this effect is of physical origin - the increasing correlation time due to appearance of long slugs (see Fig. 3d).
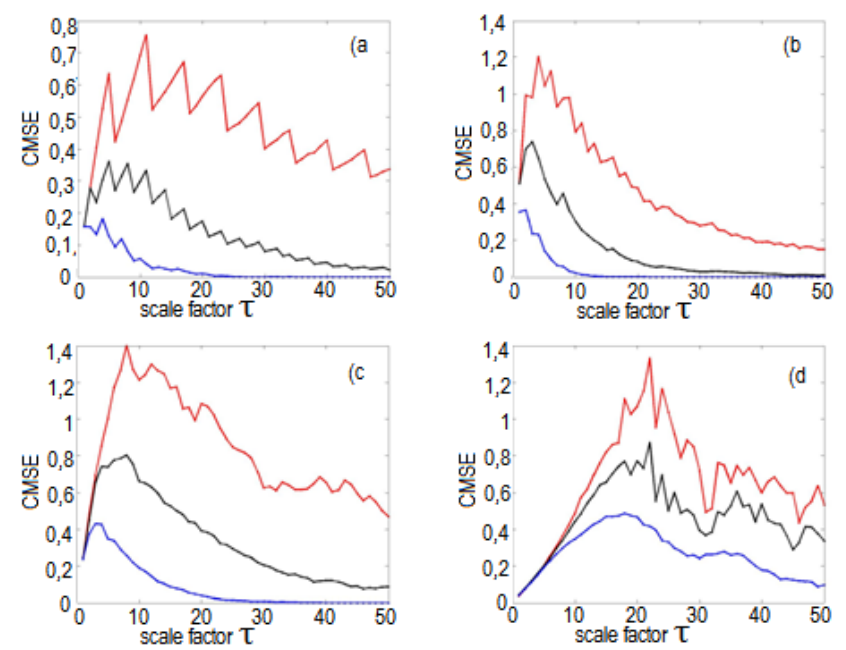

Fig. 12. The Multiscale entropy for the cases presented in Fig. 2. Colours corresponds to different similarity factors: red curve $r=0.25 \sigma(x)$, black curve $-r=0.5 \sigma(x)$, blue curve $r=1.0 \sigma(x)$ (where $\sigma(x)$ denotes the corresponding standard deviation of the original time series).
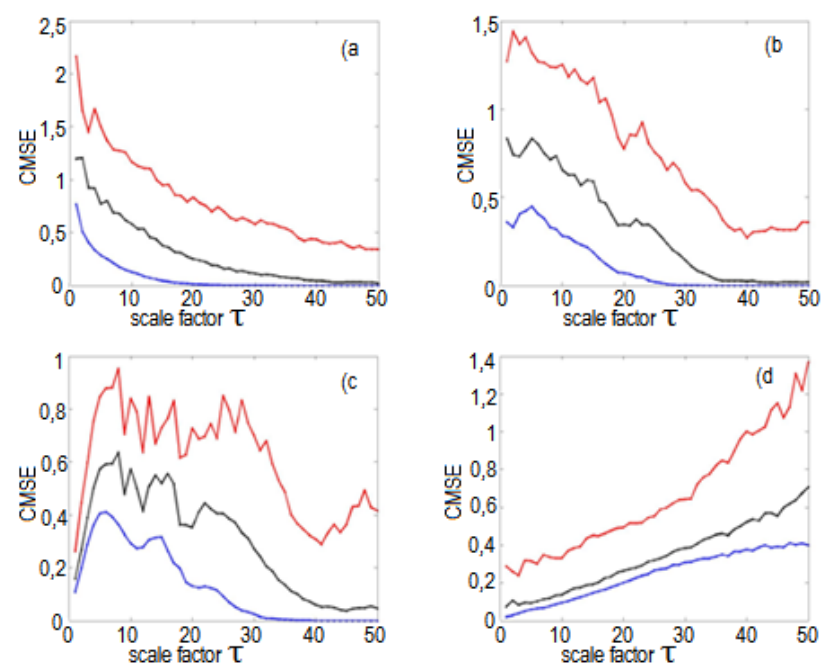

Fig. 13. The Multiscale entropy for the cases presented in Fig. 3. Colours corresponds to different similarity factors: red curve $r=0.25 \sigma(x)$, black curve $-r=0.5 \sigma(x)$, blue curve $r=1.0 \sigma(x)$ (where $\sigma(x)$ denotes the corresponding standard deviation of the original time series).

\section{CONCLUSIONS}

Analysis of time series and further application of the multiscale entropy made possible to distinguish the particular patterns of bubbles and slugs creations on the basis of multiscale entropy. The changes in patterns were caused by the stability of larger size air bubbles or slugs, which was increased for various values of flow rates of air $\left(q_{a}\right)$ and water $(q)$. In the limit of small air volume flow rate $q_{a}$, there was a disturbed noisy flow of water with small bubbles. Moreover, in this limit, the bubbles were characterized by different sizes and velocities.

For larger $q_{a}$, we identified aggregation of air bubbles of different sizes to create stable air slugs. Formation of bubbles and slugs was clearly signalled by the histograms. By means of wavelet analysis, we observed the conditions of periodic interchange in the flow of water-air mixture. Finally, slugs' appearance was accompanied by the change of negative to positive inclination in entropy. The present results based on the digital camera data are much more transparent as compared to our previous attempts, where we used the laser transmittivity time series (Gorski et al., 2015). In the previous case, increasing the scattering of the laser light occurred by passing through the owing corrugated surfaces of elongated bubbles. In this article, we focused on an automatic two-phase flow identification and related bifurcations in shape formations. Therefore, the identification of bubbles of $3 \mathrm{D}$ shapes (Mukin, 2016) is beyond this study.

\section{REFERENCES}

1. Anjos G.R., Borhani N., Mangiavacchi N., Thome J. R. (2014), A 3D moving mesh Finite Element Method for two-phase flows, Journal of Computational Physics, 270, 366-377.

2. Ansari M.R., Azadi R. (2016), Effect of diameter and axial location on upward gas-liquid two-phase flow patterns in intermediate-scale vertical tubes, Annals of Nuclear Energy, 94, 530-540.

3. Borowiec M., Rysak A., Betts D.H., Bowen C.R., Kim H.A., Litak G. (2014), Complex response of a bistable laminated plate: Multiscale entropy analysis, European Physical Journal Plus, 129, 211.

4. Borowiec M., Sen A.K., Litak G., Hunicz J., Koszalka G., Niewczas A. (2010), Vibrations of a vehicle excited by real road profiles, Forschung im Ingenieurwesen, 74, 99-109.

5. Chen L., Tian Y.S., Karayiannis T.G. (2006), The e_ect of tube diameter on vertical two-phase flow regimes in small tubes, International Journal of Heat and Mass Transfer, 49, 4220-4230.

6. Costa M., Goldberger A.L., Peng C.-K. (2005), Multiscale entropy analysis of biological signal, Physical Review, E 71, 021906.

7. Costa M., Peng C.-K., Goldberger A.L., Hausdorff J.M. (2003), Multiscale entropy analysis of human gait dynamics, Physica, A 330, 53-60.

8. Fan C., Li H., Ren X. (2015), The order recurrence quantification analysis of the characteristics of two-phase flow pattern based on multi-scale decomposition, Transactions of the Institute of Measurement and Control, 37, 793-804.

9. Fan C.-L., Jin N.-D., Chen X.-T., Gao Z.-K. (2013), Multi-scale permutation entropy, A complexity measure for discriminating twophase flow dynamics, Chinese Physics Letters, 30, 090501.

10. Gorski G., Litak G., Mosdorf R., Rysak A. (2015b), Selfaggregation phenomenon and stable flow conditions in a two-phase flow through a minichanel, Zeitschrift fuer Naturforschung, A 70, 843-849.

11. Gorski G., Litak G., Mosdorf R., Rysak A. (2016), Dynamics of a two-phase flow through a minichannel, Transition from churn to slug flow, European Physical Journal Plus, 131, 111.

12. Gorski G., Litak G., Mosdorf R., Rysak A., (2015a), Two phase flow bifurcation due to turbulence, Transition from slugs to bubbles, European Physical Journal, B 88, 239.

13. Gorski G., Litak G., Mosdorf R., Rysak R. (2015), Dynamics of two-phase flow through a minichannel, Fourier and multiscale entropy analyses, Applied Mechanics and Materials, 791, 217-223.

14. Grassberger P. (1991), Information and complexity measures in dynamical systems, In: Atmanspacher H., Scheingraber H. (Eds.), Information Dynamics, New York, Plenum Press, 15-33.

15. Haralick R.M., Srernberg S.R., Zhuang X. (1987), Image analysis using mathematical morphology, IEEE Transactions on Pattern Analysis and Machine Intelligence, PAMI-9, 532-550. 
16. Jin N.D., Nie X.B., Ren Y.Y., Liu X.B. (2003), Characterization of oil/water two-phase flow patterns based on nonlinear time series analysis, Flow Measurement and Instrumentation 14, 169-175.

17. Kumar P., Foufoula-Georgiou E. (1997), Wavelet analysis for geophysical applications, Reviews of Geophysics, 35, 385-412.

18. Lian E.Y., Ren Y.Y., Han Y.F., Liu W.X,. Jin N.D., Zhao J.Y. (2016), Multi-Scale morphological analysis of conductance signals in vertical upward gas-liquid two-phase flow, Zeitschrift fuer Naturforschung, A 71, 1031-1052.

19. Litak G., Syta A., Rusinek R. (2011), Dynamical changes during composite milling, recurrence and multiscale entropy analysis. International Journal of Advanced Manufacturing Technology, 56, 445-453.

20. Lonkwic P., Łygas K., Wolszczak P., Molski S., Litak G. (2017), Braking deceleration variability of progressive safety gears using statistical and wavelet analyses, Measurement , 110, 90-97.

21. Mosdorf R., Cheng P., Wu H.Y., Shoji M. (2005), Non-linear analyses of flow boiling in microchannels, International Journal of Heat and Mass Transfer, 48, 4667-4683.

22. Mukin R.V. (2016), Bubble reconstruction method for wiremesh sensors measurements, Exp. Fluids, 57, 133.

23. Mydlarz-Gabryk K., Pietrzak M., Troniewski L. (2014), Study on oil-water two-phase upflow in vertical pipe, Journal of Petroleum Science and Engineering, 117, 28-36.

24. Otsu N. (1979), A threshold selection method from gray-level histograms, IEEE Transactions on Systems, Man, and Cybernetics, SMC9, 62-66.

25. Riano A.B., Rodriguez I.H., Bannwart A.C., Rodriguez O.M.H. (2015), Film thickness measurement in oil-water pipe flow using image processing technique, Experimental Thermal and Fluid Science, $68,330-338$.

26. Richman JS, Moorman JR (2000), Physiological time-series analysis using approximate entropy and sample entropy. American Journal of Physiology, Heart and Circulatory Physiology, 278, H2039-H2049.
27. Rysak A., Litak G., Mosdorf R., Gorski G. (2016), Investigation of two-phase flow patterns by analysis of Eulerian space-time correlations, International Journal of Multi-phase Flow, 85, 23-37.

28. Sen A.K., Litak G., Taccani R., Radu R., (2008), Wavelet analysis of cycle-to-cycle pressure variations in an internal combustion engine Chaos, Solitons \& Fractals, 38, 886-893.

29. Torrence C., Compo G.P. (1998), A practical guide to wavelet analysis, Bull. Amer. Meteor. Soc., 79, 61-78.

30. Wang S.F., Mosdorf R., Shoji M. (2003), Nonlinear analysis on uctuation feature of two-phase flow through a T-junction, International Journal of Heat and Mass Transfer, 46, 1519-1528.

31. Wongwises S., Pipathattakul M. (2006), Flow pattern, pressure drop and void fraction of two-phase gas-liquid flow in an inclined narrow annular channel, Experimental Thermal and Fluid Science 30, 345-354.

32. Wu S.-D., Wu C.-W., Lin S.-G., Wang C.-C., Lee K.-Y. (2013), Time series analysis using composite multiscale entropy, Entropy, 15, 1069-1084.

33. Zhao L., Rezkallah K.S. (1993), Gasliquid flow patterns at microgravity conditions, International Journal Multiphase Flow, 19, 751763.

34. Zong Y.-B., Jin N.D., Wang Z.-Y., Gao Z.-K., Wang C. (2010), Nonlinear dynamic analysis of large diameter inclined oil-water two phase flow pattern, International Journal of Multiphase Flow, 36, 166-183.

Acknowledgments: The presented research was funded by the National Science Centre, Poland - the number of decision UMO.2017/27/ $\mathrm{B} / \mathrm{ST} 8 / 02905$. 